\title{
Cooperative single-atom active centers for attenuating linear scaling effect in nitrogen reduction reaction
}

\author{
Ke Ye ${ }^{\mathrm{a} \dagger}$, Min $\mathrm{Hu}^{\mathrm{a} \dagger}$, Qin-Kun Li ${ }^{\mathrm{b}}$, Yi Luo ${ }^{\mathrm{a}}$, Jun Jiang ${ }^{\mathrm{a}}$, Guozhen Zhang ${ }^{\mathrm{a}^{*}}$ \\ ${ }^{a}$ Hefei National Laboratory for Physical Sciences at the Microscale, Chinese Academy of Sciences Center for Excellence in \\ Nanoscience, School of Chemistry and Materials Science, University of Science and Technology of China, Hefei, Anhui \\ 230026, China. \\ ${ }^{\mathrm{b}}$ Department of Materials Science and NanoEngineering, Rice University, Houston, Texas, 77005, United States.
}

KEYWORDS: density-functional calculation; single-atom catalysis; scaling relations; nitrogen reduction reaction.

\begin{abstract}
Cooperative effects of adjacent active centers are critical for single-atom catalysts (SACs) as active site density matters. Yet how it affects scaling relationships in many important reactions like nitrogen reduction reaction (NRR) is underexplored. Herein we elucidate how the cooperation of two active centers can attenuate the linear scaling effect in NRR, through the firstprinciple study on 39 SACs comprised of two adjacent ( $4 \AA$ apart) four N-coordinated metal centers $\left(\mathrm{MN}_{4}\right.$ duo) embedded in graphene. Bridge-on adsorption of dinitrogen-containing species appreciably tilts the balance of adsorption of $\mathrm{N}_{2} \mathrm{H}$ and $\mathrm{NH}_{2}$ towards $\mathrm{N}_{2} \mathrm{H}$ and thus substantially loosens the restraint of scaling relations in NRR, achieving low onset potential (V) and direct $\mathrm{N} \equiv \mathrm{N}$ cleavage (Mo, Re) at room temperature, respectively. The potential of $\mathrm{MN}_{4}$ duo in NRR casts new insight into circumventing limitations of scaling relations in heterogeneous catalysis.
\end{abstract}

Heterogeneous catalysis lies at the heart of the chemical industry. ${ }^{1}$ The ideal scenario for heterogeneous catalysis, as illustrated by the Sabatier principle, seeks a delicate balance between the activation of reactants and the desorption of products. $^{2}$ Yet for most reactions involving multiple intermediates, scaling relationships between adsorption strengths of different intermediates make such a balance difficult to achieve, limiting optimization of catalysts. ${ }^{3}$ Breaking scaling relations is crucial for heterogeneous catalyst design. Nørskov and co-workers have suggested using multiple active centers to achieve it based on the first-principle calculations ${ }^{4}$, which is supported by two recent experimental studies. $^{5-6}$ However, the ambiguity, and complexity of structures of active centers in heterogeneous catalysis make it difficult to gain a straightforward and precise understanding on how catalysts could work around the restraint of scaling relations posed to reactions. A simpler catalyst model with a well-defined structure of the active center would be helpful to decipher it.

The emerging single-atom catalysts (SACs) combining the merits of both homogeneous and heterogeneous catalysts have demonstrated excellent performances in a number of different reactions. ${ }^{7-11}$ Because SACs possess simple and unified active centers with well-defined composition and coordination environments, they are deemed an ideal type of catalysts model to provide mechanistic insight into heterogeneous catalysis. Particularly, the advent of high-density SACs due to the rapid advance of synthetic technologies ${ }^{12-18}$ and precise control of inter-site distance ${ }^{19-21}$ would facilitate the study of cooperative effects of multiple active centers. The synergetic effects between adjacent active sites on heterogeneous catalysis have drawn increasing attention in experimental work. 5, 22-26 In parallel, the computational approach plays an indispensable role in dissecting mechanisms and structure-property relationships associated with SACs. ${ }^{27}$ Previously, we have conducted a firstprinciple study on cooperative communications between two neighboring active centers, and impacts of active site density on oxygen reduction reaction (ORR) and nitrogen reduction reaction (NRR) respectively. ${ }^{28-30}$, which has prompted ourself to continue the exploration of cooperative single-atom active centers.

In the present work, we employed high-density SACs as model systems to systematically investigate impacts of multiple active centers on scaling relationships between adsorption strengths of different reaction intermediates in electrocatalytic nitrogen reduction reaction (eNRR). ${ }^{4}{ }^{31}$ eNRR has been inspired by enzymatic catalysis of nitrogenase at mild conditions and regarded as a promising solution of energy-saving nitrogen 
fixation. ${ }^{31}$ Unfortunately, it is hampered by the challenge of realizing optimal activation energy $\left(E_{\mathrm{a}}\right)$ of $\mathrm{N}_{2}$ and adsorption energy $\left(\Delta E_{\text {ads }}\right)$ of nitrogen-containing intermediate species simultaneously due to the scaling relations that couple favorable (unfavorable) $E_{\text {a }}$ and unfavorable (favorable) $\Delta E_{\text {ads }}$ together. For eNRR, based on the Bell-Evans-Polanyi principle, the strong coupling between adsorption strength of $* \mathrm{NH}_{2}$ and $* \mathrm{~N}_{2} \mathrm{H}$ (* denotes adsorbed species) is in the center of scaling relations that undermine catalytic activities. ${ }^{4}$ To surpass this limitation, it requires a decoupling mechanism to allow them to be optimized independently.

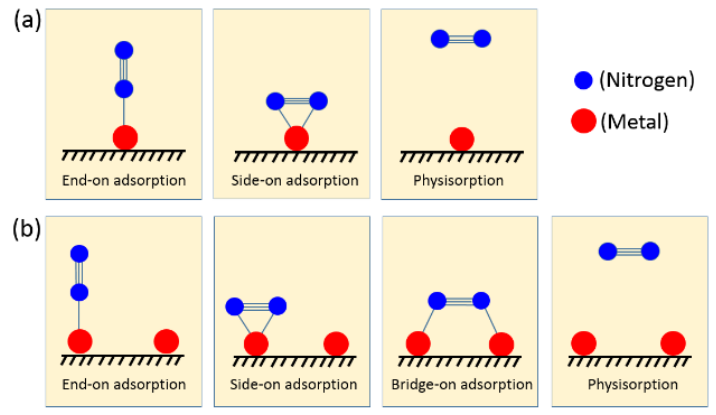

Scheme 1. Various adsorption configurations of $\mathrm{N}_{2}$ on (a) $\mathrm{MN}_{4} / \mathrm{G}$ and (b) $\mathrm{MN}_{4}$ duo/G.

Well-established eNRR mechanisms, however, resolve around associative pathways (Figure $\mathrm{S} 1)^{32}$ assuming single active center, which adsorbs dinitrogen via either end-on or side-on manners (Scheme 1). Meanwhile, alternative adsorption mode of $\mathrm{N}_{2}$ via ensemble active centers ${ }^{33}$ has largely been underexplored. Previously, we found an alternative reaction path based on cooperative bridge-on adsorption of $\mathrm{N}_{2}$ (Scheme 1) by two closely arranged $(\sim 6 \AA)$ Mo-N-C sites. ${ }^{29}$ This discovery naturally raised the question that whether the bridgeon manner might initiate a game-changing strategy of NRR. ${ }^{34}$ It then motivated us to explore in this work how the scaling relations evolve from single active center to two cooperative active centers as the bridge-on adsorption pattern of $\mathrm{N}_{2}$ and other nitrogen species are adopted.

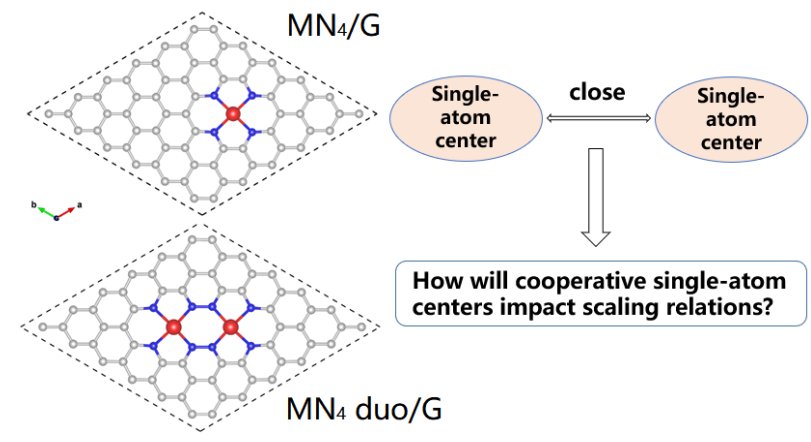

Figure 1. Top view of $\mathrm{MN}_{4} / \mathrm{G}$ and $\mathrm{MN}_{4}$ duo/G models used in this work, with the annotation of the philosophy of building them.

A single metal center coordinated with four nitrogen atoms (shorted as $\mathrm{MN}_{4}$ ) constitutes the essential moiety of many excellent molecular catalysts derived from metal porphyrins and metal phthalocyanines. ${ }^{35-37}$ The marriage of $\mathrm{MN}_{4}$ active center and two-dimensional carbon support (shorted as $\mathrm{MN}_{4} / \mathrm{C}$ ) benefits from the ease of synthesis ${ }^{13-18}$, which has generated quite a few promising SACs, including NRR catalysts ${ }^{38-43}$. As shown in Figure 1, we placed two adjacent $\mathrm{MN}_{4}$ within graphene (shorted as $\mathrm{MN}_{4} \mathrm{duo} / \mathrm{G}$ ) as a prototype model of highdensity $\mathrm{MN}_{4} \mathrm{SACs}$ to investigate how the cooperation of singleatom centers will impact scaling relations in NRR. Note that our $\mathrm{MN}_{4}$ duo model is different from other dual-atom M-N-C models $^{44-48}$ (Figure S2) in two aspects: (1) our model consists of two well-separated $\mathrm{MN}_{4}$ centers while others either have a metal-metal bond (essentially dimer) or share coordinating atoms; (2) our model focuses on cooperation between two individual active centers while others emphasize one active center with two interconnecting metal atoms.

The results and discussion are organized into two parts. In the first part, we surveyed the scaling relationship between adsorption strength of $* \mathrm{NH}_{2}$ and $* \mathrm{~N}_{2} \mathrm{H}$ in NRR occurring on 39 different $\mathrm{MN}_{4} / \mathrm{G}$ and $\mathrm{MN}_{4}$ duo/G (Figure S3), represented by their respective Gibbs adsorption free energies $\left(\Delta G^{*} \mathrm{NH}_{2}\right.$ and $\Delta G^{*} \mathrm{~N}_{2} \mathrm{H}$ ), and showed how it appreciably shifts as the adsorption mode of $* \mathrm{~N}_{2}$ and $* \mathrm{~N}_{2} \mathrm{H}$ varies from end-on to sideon to bridge-on. Equivalently, the significant changes of scaling relations for bridge-on mode were demonstrated in the form of a favorable shift of the volcano plot. In the second part, under the guidance of altered scaling relations, we investigated several potential NRR catalysts, and achieved low onset potential $(\mathrm{V})$ and direct $\mathrm{N} \equiv \mathrm{N}$ cleavage (Mo, Re) at room temperature (energy barrier $<0.85 \mathrm{eV}$ ), respectively. Our results demonstrate the importance of cooperation of monodispersed active centers, which casts new insight into the rational design of SACs for NRR at mild conditions.

\section{RESULTS AND DISCUSSION}

After building two different sets of $\mathrm{MN}_{4}$-type SACs: $\mathrm{MN}_{4} / \mathrm{G}$ and $\mathrm{MN}_{4}$ duo/G (M=transition metals and main group IIIA-VA metals), we first examined their stabilities in terms of thermodynamic stability represented by formation energy $\left(E_{\mathrm{f}}\right)$ and electrochemical stability represented by dissolution potential $\left(U_{\text {diss }}\right)$. The majority of them are found to be thermodynamically stable (See Figure S4, Table S1, and associated text in Supporting Information). Since we used the atomic energy in bulk metal as the reference state of the metal, the assignment of "unstable" in the sense of formation energy does not mean it would decompose or undergo aggregation. We expected a large energy barrier for metal migration from one site to another will prevent them from clustering. ${ }^{29}$ Further, as we explore the scaling relationship of adsorption of different intermediates of NRR, these seemly "unstable" $\mathrm{MN}_{4}$ duo models are all valuable data points that can help sketch the whole picture we need.

All possible adsorption patterns of $\mathrm{N}_{2}$ as illustrated in Scheme 1 were investigated for all $\mathrm{MN}_{4}$ and $\mathrm{MN}_{4}$ duo models. The main group metals of interest could not effectively activate 
adsorbed $\mathrm{N}_{2}$, because they invoke physisorption or very weak chemisorption. Transition metals develop five different scenarios for the adsorption of $\mathrm{N}_{2}$ (Table S2). In the rest of presentation, only $\mathrm{MN}_{4}$ and $\mathrm{MN}_{4}$ duo enabling substantial chemisorption of $\mathrm{N}_{2}$ will be examined in the study of scaling relations of NRR intermediates.

For associative NRR pathways, the first $\left(* \mathrm{~N}_{2}+\mathrm{H}^{+}+\mathrm{e}^{-} \rightarrow\right.$ $\left.* \mathrm{~N}_{2} \mathrm{H}\right)$ and sixth $\left(* \mathrm{NH}_{2}+\mathrm{H}^{+}+\mathrm{e}^{-} \rightarrow * \mathrm{NH}_{3}\right)$ reductive hydrogenation steps are the most likely potential-limiting steps. ${ }^{34}$ Their respective free energy changes $\left(\Delta G_{1}\right.$ and $\left.\Delta G_{6}\right)$ are coupled through an inversely proportional relationship. Since $\Delta G_{1}$ and $\Delta G_{6}$ scale well with $\Delta G^{*} \mathrm{~N}_{2} \mathrm{H}$ for better $\Delta G^{*} \mathrm{NH}_{2}$ respectively (Figure S5), the key scaling relations in NRR eventually fall onto $\Delta G^{*} \mathrm{~N}_{2} \mathrm{H}$ and $\Delta G^{*} \mathrm{NH}_{2}$.
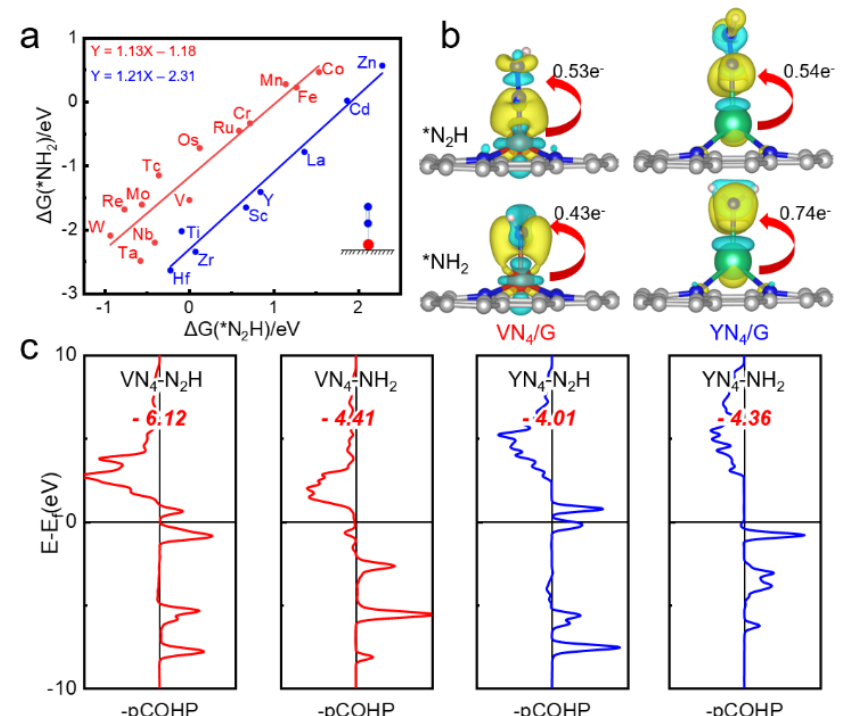

Figure 2. (a) The scaling relationship between $\Delta \mathrm{G} * \mathrm{NH}_{2}$ and $\Delta \mathrm{G} * \mathrm{NH}_{2}$ for end-on adsorption mode on $\mathrm{MN}_{4} / \mathrm{G}$. (b) The differential charge density profiles of end-on $* \mathrm{~N}_{2} \mathrm{H}$ and $* \mathrm{NH}_{2}$ by $\mathrm{VN}_{4} / \mathrm{G}$ and $\mathrm{YN}_{4} / \mathrm{G}$ with an isosurface value of $5 \times 10^{-3}$ e $\AA^{-3}$. Yellow and blue bubbles represent charge accumulation and depletion, respectively. (c) Projected crystal orbital Hamilton population (pCOHP) between the metal centers ( $\mathrm{V}$ and $\mathrm{Y}$ ) and the nitrogen adatom. The values of integrated COHP (ICOHP) are shown in red bold italics.

On $\mathrm{MN}_{4} / \mathrm{G}$, as expected, the adsorption of $\mathrm{NH}_{2}$ scales well with $\mathrm{N}_{2} \mathrm{H}$ in end-on mode (Figure 2a), in line with their scaling relationship established on pure metals surfaces. ${ }^{4}$ The transition metals can be divided into two groups based on two respective fitting lines. Group 1 (labels in blue) includes the elements in groups IB-IVB, while group 2 (labels in red) contains those in groups VB-VIIIB. (Figure S6) Note that the balance between adsorption of $\mathrm{N}_{2} \mathrm{H}$ and $\mathrm{NH}_{2}$ shifts towards $\mathrm{N}_{2} \mathrm{H}$ from group 1 to group 2, which can be interpreted by the amount of charge transfer from metal to nitrogen species (Figure 2b) based on Bader charge profile and the bonding strength between them (Figure 2c) using the integrated crystal orbital Hamilton population (ICOHP) ${ }^{49-52}$ values (more negative value indicates stronger bonding). Take $\mathrm{V}$ and $\mathrm{Y}$ for example, more negative charges transfer from $\mathrm{V}$ to $\mathrm{N}_{2} \mathrm{H}$ than to $\mathrm{NH}_{2}$, while less from $\mathrm{Y}$ to $\mathrm{N}_{2} \mathrm{H}$ than $\mathrm{NH}_{2}$; the $\mathrm{M}-\mathrm{N}$ bonding interaction is appreciably stronger in $\mathrm{MN}_{4}-\mathrm{N}_{2} \mathrm{H}$ than in $\mathrm{MN}_{4}-\mathrm{NH}_{2}$ for $\mathrm{V}$, while it's weaker in the former than the latter for $\mathrm{Y}$. The same distinction between group 1 and 2 can be found on all types of adsorption mode on both $\mathrm{MN}_{4} / \mathrm{G}$ and $\mathrm{MN}_{4}$ duo/G (Figure S7). Since preferable enhancing the adsorption of $\mathrm{N}_{2} \mathrm{H}$ over $\mathrm{NH}_{2}$ is favorable to mitigate the uphill energetics of activating the adsorbed $\mathrm{N}_{2}$ and producing $\mathrm{NH}_{3}$ from $\mathrm{NH}_{2}$, we then focus on the study of transition metals in group 2 in the rest of work.

From $\mathrm{MN}_{4} / \mathrm{G}$ to $\mathrm{MN}_{4}$ duo/G, the scaling relationships between $\Delta G^{*} \mathrm{~N}_{2} \mathrm{H}$ and $\Delta G^{*} \mathrm{NH}_{2}$ in end-on and side-on adsorption modes do not change significantly (Figure 3), as only one metal center bonds with adsorbates. However, bridge-on adsorption of $\mathrm{N}_{2} \mathrm{H}$ on $\mathrm{MN}_{4}$ duo/G appreciably alters the landscape of scaling relations (Figure 3a). As Figure 3a shows, bridge-on mode appreciably reduces the slope and shifts up the intercept of scaling line relative to end-on mode while side-on mode reduces slope at the cost of shifting down the intercept. Note that smaller slope and higher intercept values enable strengthening adsorption of $\mathrm{N}_{2} \mathrm{H}$ much more than $\mathrm{NH}_{2}$, which facilitates dinitrogen activation without impeding the final reductive hydrogenation producing ammonia. Again, the strengthening of $\mathrm{N}_{2} \mathrm{H}$ in bridge-on mode relative to end-on and side-on is demonstrated using Bader charge and ICOHP profiles (Figure 3b). Compared to end-on and side-on modes where only one metal center bond with $\mathrm{N}_{2} \mathrm{H}$, bridge-on mode has two collaborative metal centers binding to $\mathrm{N}_{2} \mathrm{H}$, which increases electrons transferred to anti-bonding orbitals of $\mathrm{N}_{2} \mathrm{H}$ and overall metal-nitrogen bonding strength.

In addition, $\Delta G 1$ barely scales with $\Delta G^{*} \mathrm{~N}_{2} \mathrm{H}$ (Figure 3c) for bridge-on mode, which is in sharp contrast to the well scaling between the two in end-on and side-on modes. Here the internal energy part plays a dominant role while the entropy factor has negligible contribution (Table S3). The change of dependence of $\Delta G 1$ on $\Delta G * \mathrm{~N}_{2} \mathrm{H}$ can be explained by the change of binding strength from $* \mathrm{~N}_{2}$ to $* \mathrm{~N}_{2} \mathrm{H}$. The integral of partial density of states (pDOS) of nitrogen atoms from $-3 \mathrm{eV}$ to $0 \mathrm{eV}$ (Fermi level is reset to 0 ) is an indicator of effective number of electrons involved in the bonding interaction between nitrogen and metal atoms. Thus, the difference of this integral between $* \mathrm{~N}_{2}$ and $* \mathrm{~N}_{2} \mathrm{H}$ roughly reflects the difference of their binding strength to metal centers. Clearly, bridge-on mode has the smallest difference while end-on and side-on bear comparable changes (Figure 3d). As two cooperative metal centers accommodate dinitrogen-containing species together, they not only bind them stronger but also mitigate the uphill energetics of first dinitrogen hydrogenation. Consequently, in bridge-on associated NRR pathways, $\Delta G 1$ and $\Delta G 6$ are decoupled (Figure S8), creating room for substantial improvement of overall catalytic activity, as seen in the volcano plots of NRR occurring on $\mathrm{MN}_{4}$ duo (Figure 4).

Based on the volcano plot associated with bridge-on adsorption mode (Figure 4c), we have identified four selected $\mathrm{MN}_{4}$ duo/G models $(\mathrm{M}=\mathrm{Mo}, \mathrm{Re}, \mathrm{V}, \mathrm{Os})$ as potential NRR catalysts. Although Tc appears to be the closest element to the 
vertex of volcano curve, it has to be excluded because it is radioactive. Then, for $\mathrm{OsN}_{4}$ duo/G, initial $\mathrm{N}_{2}$ adsorption adopts an end-on rather than bridge-on mode (Table S4). Thus, we skipped Os as well. In the rest of presentation, we mainly discussed Mo, Re, and V. Intriguingly, V, Mo, and Re are in one diagonal line in the periodic table, which may account for their similar catalytic activities in NRR. In addition to homonuclear $\mathrm{MN}_{4}$ duo/G models, we have also considered heteronuclear $\mathrm{MN}_{4}$ duo/G models in the form of hybrid $\mathrm{MN}_{4}$ duo. Therefore, we have a total of six $\mathrm{MN}_{4}$ duo/G models, including $\mathrm{VN}_{4}$ duo, $\mathrm{MoN}_{4}$ duo, $\mathrm{ReN}_{4}$ duo, $\mathrm{VN}_{4}-\mathrm{MoN}_{4}, \mathrm{MoN}_{4}$ $\mathrm{ReV}_{4}$, and $\mathrm{ReV}_{4}-\mathrm{VN}_{4}$.
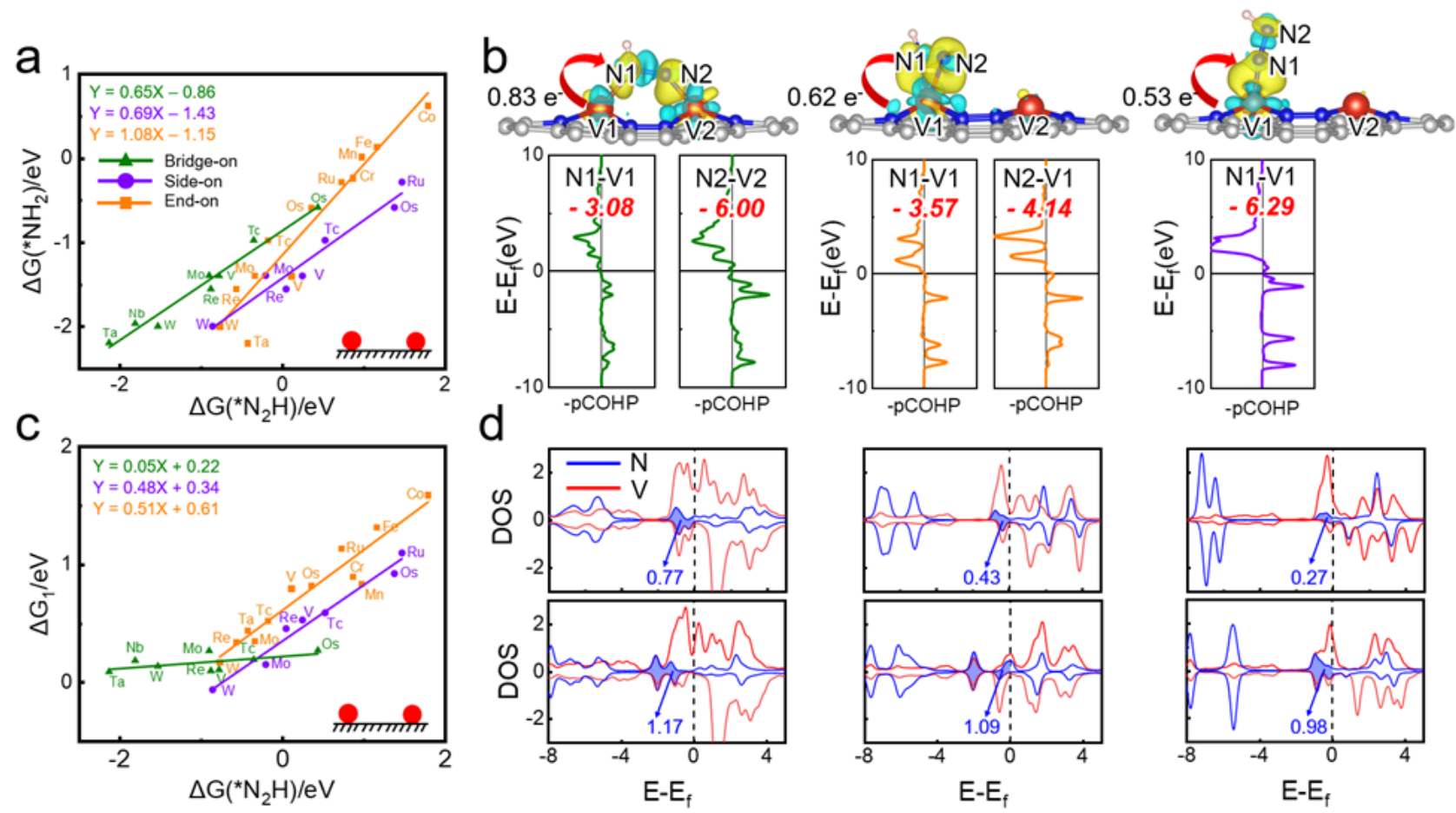

Figure 3. (a) The scaling relationships between $\Delta \mathrm{G}^{*} \mathrm{NH}_{2}$ and $\Delta \mathrm{G}^{*} \mathrm{~N}_{2} \mathrm{H}$ as $\mathrm{N}_{2} \mathrm{H}$ is adsorbed on $\mathrm{MN} \mathrm{N}_{4}$ duo/G via end-on, side-on, and bridge-on modes, respectively. (b) The differential charge densities of $* \mathrm{~N}_{2} \mathrm{H}$ in bridge-on, side-on, and end-on (from left

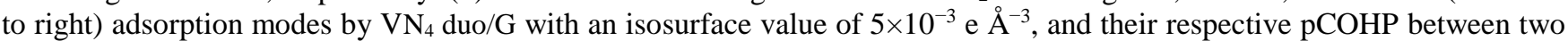
$\mathrm{V}$ atoms and the nitrogen adatoms. Yellow and blue bubbles represent charge accumulation and depletion, respectively. The values of ICOHP are shown in red bold italics. (c) The scaling relationships between $\Delta \mathrm{G}^{*} \mathrm{~N}_{2} \mathrm{H}$ and $\Delta \mathrm{G} 1$ as both $\mathrm{N}_{2}$ and $\mathrm{N}_{2} \mathrm{H}$ are adsorbed on $\mathrm{VN}_{4}$ duo/G via, respectively. (d) Electronic densities of states of $* \mathrm{~N}_{2}$ (up) and $* \mathrm{~N}_{2} \mathrm{H}$ (down) adsorbed via end-on, side-on, and bridge-on modes on $\mathrm{VN}_{4}$ duo/G, respectively.
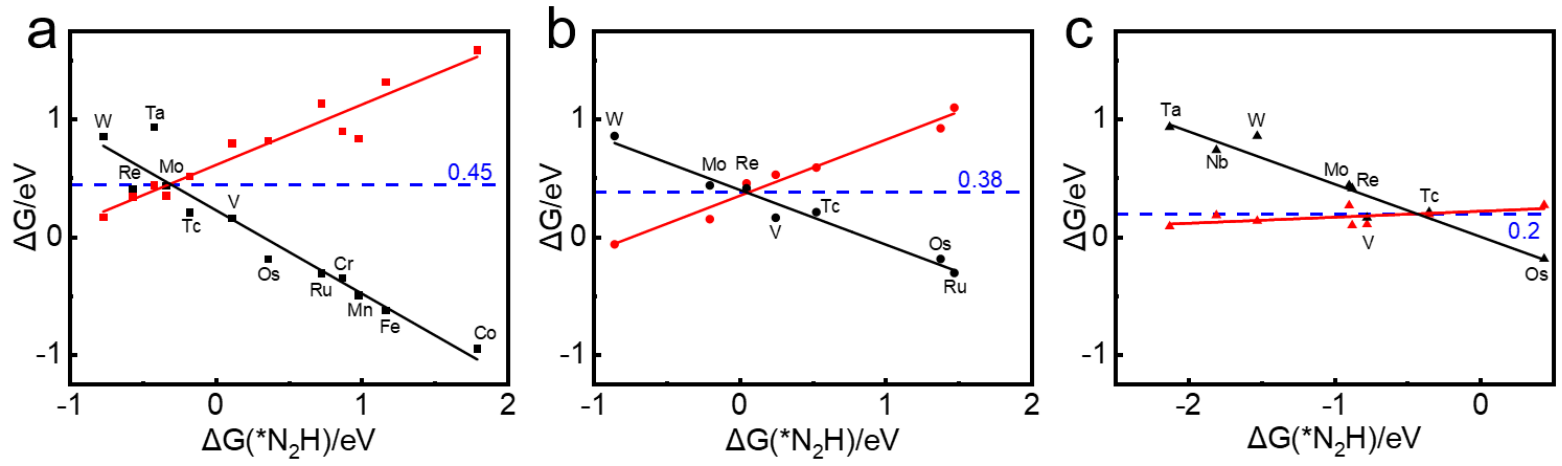

Figure 4. The scaling relationships between $\Delta \mathrm{G}_{1}$ and $\Delta \mathrm{G}^{*} \mathrm{~N}_{2} \mathrm{H}$ (red line) and between $\Delta \mathrm{G}_{6}$ and $\Delta \mathrm{G}^{*} \mathrm{~N}_{2} \mathrm{H}$ (black line) for end-on, side-on, and bridge-on (from left to right) adsorption modes of $* \mathrm{~N}_{2}$ and $* \mathrm{~N}_{2} \mathrm{H}$. The blue dash line in each panel indicates the theoretical lower bound of energetics of presumable potential-limiting steps of NRR in respective adsorption mode. 
Using these models, we calculated the full NRR pathways starting from bridge-on adsorption and obtained the onset potential of NRR that signifies the activity of the catalyst of interest. We first examined the adsorption energies of bridgeon adsorbed $\mathrm{N}_{2}$, associated charge redistribution, and geometric changes of reactant-active center complexes. As shown in Table S5, the computed binding energies are between -1.46 and -1.82 $\mathrm{eV}$ for bridge-on adsorption, accompanied by substantial charge transfer $(0.59 \sim 0.93$ |e| by Bader charge analysis $)$ from both two $\mathrm{MN}_{4}$ sites to $\mathrm{N}_{2}$. The corresponding charge distribution is also demonstrated by the differential charge densities of $\mathrm{N}_{2}-\mathrm{MN}_{4}$ duo binding complexes (Figure S9), which illustrates electron gain in the antibonding orbital of $\mathrm{N}_{2}$. As a result, the adsorbed $\mathrm{N}_{2}$ is activated, with an appreciable stretching of $\mathrm{N}-\mathrm{N}$ bond from $1.11 \AA$ (free $\mathrm{N}_{2}$ in the gas phase) to $1.18 \sim 1.23 \AA$.

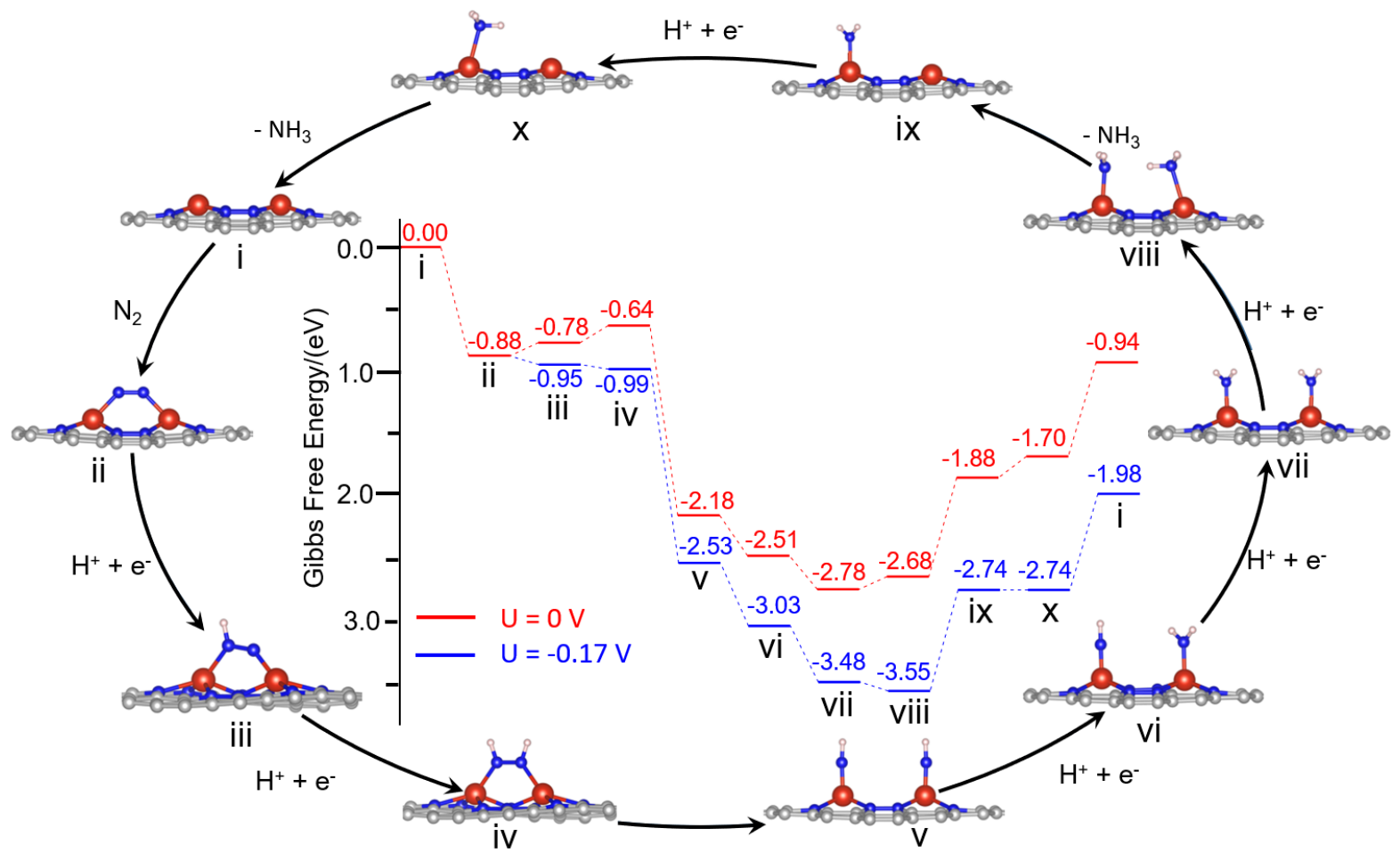

Figure 5. The reaction profile of the bridge-on adsorption initiated0020030NRR pathway on VN4 duo/G model. Roman numbers denote all reaction species along the pathway.

Then we investigated the $\mathrm{N} \equiv \mathrm{N}$ bond dissociation following the bridge-on adsorption. The transition state structures are showing in Figure S10. Remarkably, 3 out of 6 selected models, including $\mathrm{MoN}_{4}$ duo, $\mathrm{ReN}_{4}$ duo, and $\mathrm{MoN}_{4}-\mathrm{ReN}_{4}$, enable direct $\mathrm{N} \equiv \mathrm{N}$ bond breaking with a reaction barrier below $0.91 \mathrm{eV}$ (Table S6), a well-documented threshold for chemical reactions available at room temperature. ${ }^{53}$ This unusual phenomenon suggests a dissociative NRR mechanism, in sharp contrast to common $\mathrm{N} \equiv \mathrm{N}$ bond dissociation aided by reductive hydrogenation seen in associative NRR pathways. Intriguingly, this pathway will avoid the formation of $* \mathrm{~N}_{2} \mathrm{H}$, one of two key intermediate species in NRR, suggesting the possibility of circumventing the well-established scaling relationship for $\mathrm{NRR}$ by direct $\mathrm{N} \equiv \mathrm{N}$ bond dissociation through the joint effort of two adjacent active centers.

To understand it, we adopted the $\mathrm{N}-\mathrm{N}$ distance as the reaction coordinate to understand the trend of energy barriers of $\mathrm{N}-\mathrm{N}$ dissociation. Intriguingly, the barriers are positively correlated with the distances (Figure S11-a), because a smaller difference of N-N distance between the transition state (TS) and initial state will result in a smaller barrier for $\mathrm{N}-\mathrm{N}$ bond dissociation. This is typical for an early TS, whose structure is close to the corresponding initial state. Then we examined the relationship between the stabilities of all six models and the corresponding energy barrier of TS and found that the lower stability of the catalyst results in a smaller energy barrier (Figure S11-b). Since the $\mathrm{ReN}_{4}$ duo is the least stable one among these six models, it has the lowest direct $\mathrm{N}_{2}$ dissociation barrier. Similarly, $\mathrm{MoN}_{4}$ duo and $\mathrm{MoN}_{4}-\mathrm{ReN}_{4}$ enables direct $\mathrm{N}_{2}$ dissociation at room temperature for their relative lability. The direct $\mathrm{N} \equiv \mathrm{N}$ breaking aided by bridge-on adsorption may open a new possibility of breaking scaling relationships applied on NRR, which will be investigated in the future. In contrast, as the stabilities increase, which are the cases of $\mathrm{VN}_{4}$ duo, $\mathrm{VN}_{4}-\mathrm{MoN}_{4}$, and $\mathrm{VN}_{4}-\mathrm{ReN}_{4}$, the dissociative pathway is unfavorable at room temperature; thus, NRR will proceed via associative mechanisms in which $\mathrm{N} \equiv \mathrm{N}$ breaking takes place after one or two steps of reductive hydrogenation. Their bridge-on adsorption initiated NRR pathways is similar to the one occurring on $\mathrm{MoN}_{3}$ duo 
embedded in graphene. ${ }^{29}$ The balance between stability and lability of these catalysts may be a key factor for determining the preferred reaction pathways of NRR occurring on them.

The full reaction coordinates of NRR with all six of these models are collected in Figure S12 through Figure S17. Based on the Gibbs free energy data therein, we found that $\mathrm{VN}_{4} \mathrm{duo} / \mathrm{G}$ possesses the lowest limiting potential $(0.18 \mathrm{~V})$ and thus bears the highest theoretical activity. The reaction coordinates are shown in Figure 5. For the strong adsorption, the $\Delta G * N_{2} \rightarrow * N_{2} H$ for bridge-on $* \mathrm{~N}_{2}$ is $0.11 \mathrm{eV}$ and the $\mathrm{N}-\mathrm{N}$ distance is stretched to $1.31 \AA$. The second reductive hydrogenation $(* \mathrm{NNH} \rightarrow$ $* \mathrm{HNNH})$ is endothermic $\left(\Delta \mathrm{G} * \mathrm{~N}_{2} \mathrm{H} \rightarrow * \mathrm{HNNH}=0.16 \mathrm{eV}\right)$, with the $\mathrm{N}-\mathrm{N}$ distance of $1.37 \AA$. Intriguingly, this bridge-on $* \mathrm{HNNH}$ resembles the bridging "diazene" species found in nitrogenase. ${ }^{54-55}$ Then $*$ HNNH dissociates and produces two $* \mathrm{NH}$, each of which is attached to one $\mathrm{V}$ atom. The energy barrier $(0.78 \mathrm{eV})$ for this $\mathrm{N} \equiv \mathrm{N}$ bond breaking can be easily overcome at room temperature. Then the two *NH alternately undergo reductive hydrogenation steps, producing two $* \mathrm{NH}_{2}$. Both reductive hydrogenation of $* \mathrm{NH}_{2}$ and desorption of $\mathrm{NH}_{3}$ from $* \mathrm{NH}_{3}$ are thermodynamically uphill. The electrochemical step can be promoted only by applying an external potential. At $\mathrm{U}=-0.17 \mathrm{~V}, \Delta \mathrm{G} * \mathrm{NH}_{2} \rightarrow * \mathrm{NH}_{3}$ becomes zero, and the uphill energetics is substantially mitigated (Figure 5). This remarkably low limiting potential benefits from the cooperation of two adjacent $\mathrm{VN}_{4}$ sites that help breaking the scaling relationship of NRR by properly balancing the adsorption energies of $* \mathrm{~N}_{2} \mathrm{H}$ and $* \mathrm{NH}_{2}$. For the endergonic $(\sim 0.7 \mathrm{eV})$ desorption of ammonia, we expect that the solvation of ammonia can mitigate the unfavorable energetics because it will stabilize the desorbed ammonia. 4 , 39, 43, 56-57 Thus, $\mathrm{NH}_{3}$ desorption may not be a main obstacle in NRR and, hence, is not considered in detail here.

\section{CONCLUSION}

In this work, we have conducted a systematic first-principle study on impacts of bridge-on $\mathrm{N}_{2}$ adsorption by two adjacent $\mathrm{MN}_{4}$ sites on scaling relationships within electrocatalytic NRR and found the key to attenuate the scaling relations is to create inhomogeneous adsorption between dinitrogen-containing species and nitrogen-containing species.

We confirmed that the scaling relationship between adsorption of $\mathrm{N}_{2} \mathrm{H}$ and $\mathrm{NH}_{2}$ hold for NRR occurring on single active center, regardless of $\mathrm{MN}_{4}$ or $\mathrm{MN}_{4}$ duo. Since the room of improvement of NRR activity associated with one active center is limited, we have to go beyond conventional end-on and sideon adsorption modes. Intriguingly, the side-on fashion benefits from different metal-nitrogen bonding patterns for $* \mathrm{~N}_{2} \mathrm{H}$ and $* \mathrm{NH}_{2}$, hinting a path for the evolution of NRR catalysts. ${ }^{43-44,58}$ The bridge-on adsorption mode can be viewed as a two-center version of "side-on". It further widens the gap of metal-nitrogen bonding strengths between $* \mathrm{~N}_{2} \mathrm{H}$ and $* \mathrm{NH}_{2}$, enabling us to decouple the optimization of $\left(* \mathrm{~N}_{2}+\mathrm{e}^{-}+\mathrm{H}^{+} \rightarrow * \mathrm{~N}_{2} \mathrm{H}\right)$ and $\left(* \mathrm{NH}_{2}+\mathrm{e}^{-}+\mathrm{H}^{+} \rightarrow * \mathrm{NH}_{3}\right)$. Thus, it shows the potential of making substantial progress towards breaking scaling relations in NRR. Importantly, the bridge-on pattern relies on the proximity of two metal active sites, which could well explain other reported promising NRR catalytic systems invoking bridge-on like activation of $\mathrm{N}_{2}$ by multiple active centers. ${ }^{59-61}$

Based on the survey, we then identified $\mathrm{VN}_{4}$ duo and $\mathrm{ReN}_{4}$ duo as promising NRR catalysts through associative mechanisms and dissociative mechanisms, respectively. For $\mathrm{VN}_{4}$ duo, the remarkably low onset potential $(0.18 \mathrm{eV})$ indicates the level of excellent activity of NRR that can be achieved by high-density SACs. For $\mathrm{ReN}_{4}$ duo, the most appealing feature is the low energy barrier $(0.52 \mathrm{eV})$ of direct $\mathrm{N} \equiv \mathrm{N}$ bond dissociation enabling a dissociative mechanism for NRR at room temperature, which for sure will inspire more mechanistic study on dissociative pathways with multiple concerted active centers. We also expect it motivates more exploration on how the delicate balance between stability and lability of active centers would affect the dynamics of $\mathrm{N}_{2}$ activation process.

In summary, through a comprehensive understanding of scaling relationships for $\mathrm{NRR}$ in $\mathrm{MN}_{4}$-type single-atom catalysts, we found cooperation of two adjacent single-atom active centers can attenuate scaling relation effect and facilitate nitrogen fixation. Although our computational models are based on ideal reaction conditions, we expect our work casting new light on NRR mechanisms with collective active centers will be helpful for the rational design of high-density SACs and prompting mechanistic study on breaking scaling relationships existing in other reactions (e.g. $\mathrm{CO}_{2}$ reduction ${ }^{62}$ and oxygen reduction reaction) aided by cooperative multiple active centers. We will also continue the investigation of cooperative single-atom active centers under more realistic reaction conditions in the future work.

\section{COMPUTATIONAL DETAILS}

All spin-polarized density functional theory (DFT) calculations were performed using the Perdew-BurkeErnzerhof $(\mathrm{PBE})^{63}$ functional in conjunction with plane-wave projected augmented wave $(\mathrm{PAW})^{64}$ method as implemented in Vienna ab initio simulation program (VASP) ${ }^{65-66}$. The kinetic cutoff energy for the plane-wave basis set was set to be $480 \mathrm{eV}$. The Gaussian smearing method was adopted with a width of 0.1 $\mathrm{eV}$ to describe partial occupancies of each orbital. The first Brillouin zone was sampled by a Monkhorst-Pack scheme with a $3 \times 3 \times 1 k$-point grid. To avoid the interaction between two periodic units, a vacuum space exceeds $15 \AA$ was employed. Structures were fully relaxed until the forces were converged to less than $0.02 \mathrm{eV}^{-1}$. The Grimme's D3 dispersion correction scheme was used to describe the van der Waals interaction. The solvent effect on scaling relations are not taken into account as a previous study showed that no significant change that can alter the scaling relations has been found as solvent effect was incorporated. ${ }^{67}$ More details regarding adopted computational models, calculation of various energy values and selection of energetic descriptors are given in the Supporting Information.

\section{ASSOCIATED CONTENT}

\section{Supporting Information}


Computational detail, geometrical structures and stability validation of $\mathrm{MN}_{4}$ and $\mathrm{MN}_{4}$ duo models, the adsorption energies of $\mathrm{N}_{2}$, differential charge densities, various scaling relationships and Gibbs free energy diagrams for NRR occurring on selected $\mathrm{MN}_{4}$ duo models.

\section{AUTHOR INFORMATION Corresponding Author}

*Guozhen Zhang, Email: guozhen@ustc.edu.cn

\section{ORCID}

Guozhen Zhang: 0000-0003-0125-9666

Jun Jiang: 0000-0002-6116-5605

\section{Author Contributions}

${ }^{\dagger} \mathrm{K} . \mathrm{Y}$. and M.H. contributed equally to this work.

\section{Note}

The authors declare no competing financial support.

\section{ACKNOWLEDGMENTS}

This work was financially supported by the Ministry of Science and Technology of the People's Republi0063 of China (No. 2018YFA0208702, 2018YFA0208603, and 2017YFA0303500) and the National Natural Science Foundation of China (21703221, 21790351, and 21633006). The Supercomputing Center of University of Science and Technology of China is acknowledged for the computing resource.

\section{REFERENCES}

(1) Nørskov, J. K.; Studt, F.; Abild-Pedersen, F.; Bligaard, T. Fundamental Concepts in Heterogeneous Catalysis; John Wiley \& Sons, Inc., 2014

(2) Medford, A. J.; Vojvodic, A.; Hummelshøj, J. S.; Voss, J.; AbildPedersen, F.; Studt, F.; Bligaard, T.; Nilsson, A.; Nørskov, J. K. From the Sabatier Principle to a Predictive Theory of Transition-Metal Heterogeneous Catalysis. J. Catal. 2015, 328, 36-42.

(3) Zhao, Z.-J.; Liu, S.; Zha, S.; Cheng, D.; Studt, F.; Henkelman, G.; Gong, J. Theory-Guided Design of Catalytic Materials Using Scaling Relationships and Reactivity Descriptors. Nat. Rev. Mater. 2019, 4, 792-804.

(4) Montoya, J. H.; Tsai, C.; Vojvodic, A.; Norskov, J. K. The Challenge of Electrochemical Ammonia Synthesis: A New Perspective on the Role of Nitrogen Scaling Relations. Chemsuschem 2015, 8, 2180-6.

(5) Wang, P.; Chang, F.; Gao, W.; Guo, J.; Wu, G.; He, T.; Chen, P. Breaking Scaling Relations to Achieve Low-Temperature Ammonia Synthesis through Lih-Mediated Nitrogen Transfer and Hydrogenation. Nat. Chem. 2017, 9, 64-70.
(6) Mao, C.; Wang, J.; Zou, Y.; Qi, G.; Yang Loh, J. Y.; Zhang, T.; Xia, M.; Xu, J.; Deng, F.; Ghoussoub, M.; Kherani, N. P.; Wang, L.; Shang, H.; Li, M.; Li, J.; Liu, X.; Ai, Z.; Ozin, G. A.; Zhao, J.; Zhang, L. Hydrogen Spillover to Oxygen Vacancy of $\mathrm{TiO}_{2-\mathrm{x}} \mathrm{H}_{\mathrm{y}} / \mathrm{Fe}$ : Breaking the Scaling Relationship of Ammonia Synthesis. J. Am. Chem. Soc. 2020, 142, 1740317412 .

(7) Qiao, B.; Wang, A.; Yang, X.; Allard, L. F.; Jiang, Z.; Cui, Y.; Liu, J.; $\mathrm{Li}$, J.; Zhang, T. Single-Atom Catalysis of Co Oxidation Using $\mathrm{Pt}_{1} / \mathrm{FeO}_{\mathrm{x}}$ Nat. Chem. 2011, 3, 634-41.

(8) Cui, X.; Li, W.; Ryabchuk, P.; Junge, K.; Beller, M. Bridging Homogeneous and Heterogeneous Catalysis by Heterogeneous SingleMetal-Site Catalysts. Nat. Catal. 2018, 1, 385-397.

(9) Wang, A.; Li, J.; Zhang, T. Heterogeneous Single-Atom Catalysis. Nat. Rev. Chem. 2018, 2, 65-81.

(10) Mitchell, S.; Perez-Ramirez, J. Single Atom Catalysis: A Decade of Stunning Progress and the Promise for a Bright Future. Nat. Commun. 2020, 11,4302 .

(11) Zhuo, H. Y.; Zhang, X.; Liang, J. X.; Yu, Q.; Xiao, H.; Li, J. Theoretical Understandings of Graphene-Based Metal Single-Atom Catalysts: Stability and Catalytic Performance. Chem. Rev. 2020, 120, 12315-12341.

(12) Wan, X.; Liu, X.; Li, Y.; Yu, R.; Zheng, L.; Yan, W.; Wang, H.; Xu, M.; Shui, J. Fe-N-C Electrocatalyst with Dense Active Sites and Efficient Mass Transport for High-Performance Proton Exchange Membrane Fuel Cells. Nat. Catal. 2019, 2, 259-268.

(13) Yin, P.; Yao, T.; Wu, Y.; Zheng, L.; Lin, Y.; Liu, W.; Ju, H.; Zhu, J.; Hong, X.; Deng, Z.; Zhou, G.; Wei, S.; Li, Y. Single Cobalt Atoms with Precise N-Coordination as Superior Oxygen Reduction Reaction Catalysts. Angew. Chem. Int. Ed. 2016, 55, 10800-5.

(14) Chen, Y.; Ji, S.; Wang, Y.; Dong, J.; Chen, W.; Li, Z.; Shen, R.; Zheng, L.; Zhuang, Z.; Wang, D.; Li, Y. Isolated Single Iron Atoms Anchored on N-Doped Porous Carbon as an Efficient Electrocatalyst for the Oxygen Reduction Reaction. Angew. Chem. Int. Ed. 2017, 56, 6937-6941.

(15) Wu, J.; Zhou, H.; Li, Q.; Chen, M.; Wan, J.; Zhang, N.; Xiong, L.; Li, S.; Xia, B. Y.; Feng, G. Densely Populated Isolated Single Co-N Site for Efficient Oxygen Electrocatalysis. Adv. Energy Mater. 2019, 9, 1900149.

(16) Bauer, G.; Ongari, D.; Tiana, D.; Gaumann, P.; Rohrbach, T.; Pareras, G.; Tarik, M.; Smit, B.; Ranocchiari, M. Metal-Organic Frameworks as Kinetic Modulators for Branched Selectivity in Hydroformylation. Nat. Commun. 2020, 11, 1059.

(17) Liu, K.; Zhao, X.; Ren, G.; Yang, T.; Ren, Y.; Lee, A. F.; Su, Y.; Pan, X.; Zhang, J.; Chen, Z.; Yang, J.; Liu, X.; Zhou, T.; Xi, W.; Luo, J.; Zeng, C.; Matsumoto, H.; Liu, W.; Jiang, Q.; Wilson, K.; Wang, A.; Qiao, B.; Li, W.; Zhang, T. Strong Metal-Support Interaction Promoted Scalable Production of Thermally Stable Single-Atom Catalysts. Nat. Commun. 2020, 11, 1263 .

(18) Xiong, Y.; Sun, W.; Xin, P.; Chen, W.; Zheng, X.; Yan, W.; Zheng, L.; 
Dong, J.; Zhang, J.; Wang, D.; Li, Y. Gram-Scale Synthesis of HighLoading Single-Atomic-Site Fe Catalysts for Effective Epoxidation of Styrene. Adv. Mater. 2020, e2000896.

(19) Wu, J.; Xiong, L.; Zhao, B.; Liu, M.; Huang, L. Densely Populated Single Atom Catalysts. Small Methods 2019, 4, 1900540.

(20) He, Z.; He, K.; Robertson, A. W.; Kirkland, A. I.; Kim, D.; Ihm, J.; Yoon, E.; Lee, G. D.; Warner, J. H. Atomic Structure and Dynamics of Metal Dopant Pairs in Graphene. Nano Lett. 2014, 14, 3766-72.

(21) Lin, Y. C.; Teng, P. Y.; Yeh, C. H.; Koshino, M.; Chiu, P. W.; Suenaga, K. Structural and Chemical Dynamics of Pyridinic-Nitrogen Defects in Graphene. Nano Lett. 2015, 15, 7408-13.

(22) Li, H.; Wang, L.; Dai, Y.; Pu, Z.; Lao, Z.; Chen, Y.; Wang, M.; Zheng, X.; Zhu, J.; Zhang, W.; Si, R.; Ma, C.; Zeng, J. Synergetic Interaction between Neighbouring Platinum Monomers in $\mathrm{CO}_{2}$ Hydrogenation. Nat. Nanotechnol. 2018, 13, 411-417.

(23) Zou, N.; Zhou, X.; Chen, G.; Andoy, N. M.; Jung, W.; Liu, G.; Chen, P. Cooperative Communication within and between Single Nanocatalysts. Nat. Chem. 2018, 10, 607-614.

(24) Jiao, J.; Lin, R.; Liu, S.; Cheong, W. C.; Zhang, C.; Chen, Z.; Pan, Y.; Tang, J.; Wu, K.; Hung, S. F.; Chen, H. M.; Zheng, L.; Lu, Q.; Yang, X.; Xu, B.; Xiao, H.; Li, J.; Wang, D.; Peng, Q.; Chen, C.; Li, Y. Copper Atom-Pair Catalyst Anchored on Alloy Nanowires for Selective and Efficient Electrochemical Reduction of $\mathrm{CO}_{2}$. Nat. Chem. 2019, 11, 222-228.

(25) Fu, J.; Dong, J.; Si, R.; Sun, K.; Zhang, J.; Li, M.; Yu, N.; Zhang, B.; Humphrey, M. G.; Fu, Q.; Huang, J. Synergistic Effects for Enhanced Catalysis in a Dual Single-Atom Catalyst. ACS Catal. 2021, 11, 1952-1961.

(26) Tang, Y.; Wei, Y.; Wang, Z.; Zhang, S.; Li, Y.; Nguyen, L.; Li, Y.; Zhou, Y.; Shen, W.; Tao, F. F.; Hu, P. Synergy of Single-Atom Ni1 and Ru1 Sites on Ceo2 for Dry Reforming of Ch4. J. Am. Chem. Soc. 2019, 141, 7283-7293.

(27) Zhang, X.; Chen, A.; Chen, L.; Zhou, Z. 2d Materials Bridging Experiments and Computations for Electro/Photocatalysis. Adv. Energy Mater. 2021

(28) Li, Q.-K.; Li, X.-F.; Zhang, G.; Jiang, J. Cooperative Spin Transition of Monodispersed $\mathrm{FeN}_{3}$ Sites within Graphene Induced by $\mathrm{CO}$ Adsorption. J. Am. Chem. Soc. 2018, 140, 15149-15152.

(29) Ye, K.; Hu, M.; Li, Q. K.; Han, Y.; Luo, Y.; Jiang, J.; Zhang, G. Cooperative Nitrogen Activation and Ammonia Synthesis on Densely Monodispersed Mo-N-C Sites. J. Phys. Chem. Lett. 2020, 11, 3962-3968.

(30) Han, Y.; Li, Q. K.; Ye, K.; Luo, Y.; Jiang, J.; Zhang, G. Impact of Active Site Density on Oxygen Reduction Reactions Using Monodispersed Fe-N-C Single-Atom Catalysts. ACS Appl. Mater. Interfaces 2020, 12, 15271-15278

(31) Chen, J. G.; Crooks, R. M.; Seefeldt, L. C.; Bren, K. L.; Bullock, R. M.; Darensbourg, M. Y.; Holland, P. L.; Hoffman, B.; Janik, M. J.; Jones, A. K.; Kanatzidis, M. G.; King, P.; Lancaster, K. M.; Lymar, S. V.; Pfromm, P.; Schneider, W. F.; Schrock, R. R. Beyond Fossil Fuel-Driven Nitrogen
Transformations. Science 2018, 360 .

(32) Honkala, K.; Hellman, A.; Remediakis, I.; Logadottir, A.; Carlsson, A.; Dahl, S.; Christensen, C. H.; Nørskov, J. K. Ammonia Synthesis from First-Principles Calculations. Science 2005, 307, 555-558.

(33) Jeong, H.; Lee, G.; Kim, B. S.; Bae, J.; Han, J. W.; Lee, H. Fully Dispersed Rh Ensemble Catalyst to Enhance Low-Temperature Activity. $J$. Am. Chem. Soc. 2018, 140, 9558-9565.

(34) Qing, G.; Ghazfar, R.; Jackowski, S. T.; Habibzadeh, F.; Ashtiani, M. M.; Chen, C. P.; Smith, M. R., 3rd; Hamann, T. W. Recent Advances and Challenges of Electrocatalytic $\mathrm{N}_{2}$ Reduction to Ammonia. Chem. Rev. 2020, 120, 5437-5516.

(35) Wang, Y.; Yuan, H.; Li, Y.; Chen, Z. Two-Dimensional IronPhthalocyanine (Fe-Pc) Monolayer as a Promising Single-Atom-Catalyst for Oxygen Reduction Reaction: A Computational Study. Nanoscale 2015, 7, 11633-41.

(36) Melville, O. A.; Lessard, B. H.; Bender, T. P. Phthalocyanine-Based Organic Thin-Film Transistors: A Review of Recent Advances. ACS Appl. Mater. Interfaces 2015, 7, 13105-18.

(37) Maitarad, P.; Namuangruk, S.; Zhang, D.; Shi, L.; Li, H.; Huang, L.; Boekfa, B.; Ehara, M. Metal-Porphyrin: A Potential Catalyst for Direct Decomposition of $\mathrm{N}_{2} \mathrm{O}$ by Theoretical Reaction Mechanism Investigation. Environ. Sci. Technol. 2014, 48, 7101-10.

(38) Li, X. F.; Li, Q. K.; Cheng, J.; Liu, L.; Yan, Q.; Wu, Y.; Zhang, X. H.; Wang, Z. Y.; Qiu, Q.; Luo, Y. Conversion of Dinitrogen to Ammonia by Fen3-Embedded Graphene. J. Am. Chem. Soc. 2016, 138, 8706-9.

(39) Zhao, J.; Chen, Z. Single Mo Atom Supported on Defective Boron Nitride Monolayer as an Efficient Electrocatalyst for Nitrogen Fixation: A Computational Study. J. Am. Chem. Soc. 2017, 139, 12480-12487.

(40) Han, L.; Liu, X.; Chen, J.; Lin, R.; Liu, H.; Lu, F.; Bak, S.; Liang, Z.; Zhao, S.; Stavitski, E.; Luo, J.; Adzic, R. R.; Xin, H. L. Atomically Dispersed Molybdenum Catalysts for Efficient Ambient Nitrogen Fixation. Angew. Chem. Int. Ed. 2019, 58, 2321-2325.

(41) Tao, H.; Choi, C.; Ding, L.-X.; Jiang, Z.; Han, Z.; Jia, M.; Fan, Q.; Gao, Y.; Wang, H.; Robertson, A. W.; Hong, S.; Jung, Y.; Liu, S.; Sun, Z. Nitrogen Fixation by Ru Single-Atom Electrocatalytic Reduction. Chem 2019, 5, 204-214.

(42) Zhao, W.; Zhang, L.; Luo, Q.; Hu, Z.; Zhang, W.; Smith, S.; Yang, J. Single Mo1(Cr1) Atom on Nitrogen-Doped Graphene Enables Highly Selective Electroreduction of Nitrogen into Ammonia. ACS Catal. 2019, 9 , 3419-3425.

(43) Choi, C.; Back, S.; Kim, N.-Y.; Lim, J.; Kim, Y.-H.; Jung, Y. Suppression of Hydrogen Evolution Reaction in Electrochemical N2 Reduction Using Single-Atom Catalysts: A Computational Guideline. ACS Catal. 2018, 8, 7517-7525.

(44) Guo, X.; Gu, J.; Lin, S.; Zhang, S.; Chen, Z.; Huang, S. Tackling the Activity and Selectivity Challenges of Electrocatalysts toward the Nitrogen Reduction Reaction Via Atomically Dispersed Biatom Catalysts. J. Am. 
Chem. Soc. 2020, 142, 5709-5721.

(45) Wang, X.; Qiu, S.; Feng, J.; Tong, Y.; Zhou, F.; Li, Q.; Song, L.; Chen, S.; Wu, K. H.; Su, P.; Ye, S.; Hou, F.; Dou, S. X.; Liu, H. K.; Max Lu, G. Q.; Sun, C.; Liu, J.; Liang, J. Confined Fe-Cu Clusters as Sub-Nanometer Reactors for Efficiently Regulating the Electrochemical Nitrogen Reduction Reaction. Adv. Mater. 2020, 32, e2004382.

(46) He, T.; Puente Santiago, A. R.; Du, A. Atomically Embedded Asymmetrical Dual-Metal Dimers on N-Doped Graphene for UltraEfficient Nitrogen Reduction Reaction. J. Catal. 2020, 388, 77-83.

(47) Deng, T.; Cen, C.; Shen, H.; Wang, S.; Guo, J.; Cai, S.; Deng, M. Atom-Pair Catalysts Supported by N-Doped Graphene for the Nitrogen Reduction Reaction: D-Band Center-Based Descriptor. J. Phys. Chem. Lett. 2020, 11, 6320-6329.

(48) Lv, X.; Wei, W.; Huang, B.; Dai, Y.; Frauenheim, T. High-Throughput Screening of Synergistic Transition Metal Dual-Atom Catalysts for Efficient Nitrogen Fixation. Nano Lett. 2021, 21, 1871-1878.

(49) Deringer, V. L.; Tchougreeff, A. L.; Dronskowski, R. Crystal Orbital Hamilton Population (COHP) Analysis as Projected from Plane-Wave Basis Sets. J. Phys. Chem. A 2011, 115, 5461-6.

(50) Maintz, S.; Deringer, V. L.; Tchougreeff, A. L.; Dronskowski, R. Analytic Projection from Plane-Wave and Paw Wavefunctions and Application to Chemical-Bonding Analysis in Solids. J. Comput. Chem. 2013, 34, 2557-67.

(51) Maintz, S.; Deringer, V. L.; Tchougreeff, A. L.; Dronskowski, R. Lobster: A Tool to Extract Chemical Bonding from Plane-Wave Based Dft. J. Comput. Chem. 2016, 37, 1030-5.

(52) Nelson, R.; Ertural, C.; George, J.; Deringer, V. L.; Hautier, G.; Dronskowski, R. Lobster: Local Orbital Projections, Atomic Charges, and Chemical-Bonding Analysis from Projector-Augmented-Wave-Based Density-Functional Theory. J. Comput. Chem. 2020, 41, 1931-1940.

(53) Young, D. C., A Practical Guide for Applying Techniques to Real World Problems, John Wiley \& Sons, Inc., New York, 1st edn, 2001.

(54) Saouma, C. T.; Kinney, R. A.; Hoffman, B. M.; Peters, J. C., Transformation of an $\left[\mathrm{Fe}\left(\eta^{2}-\mathrm{N}_{2} \mathrm{H}_{3}\right)\right]^{+}$Species to Pi-Delocalized $\left[\mathrm{Fe}_{2}(\mu-\right.$ $\left.\left.\mathrm{N}_{2} \mathrm{H}_{2}\right)\right]^{2+/+}$ complexes. Angew. Chem. Int. Ed. 2011, 50, 3446-9.

(55) Hoffman, B. M.; Lukoyanov, D.; Yang, Z. Y.; Dean, D. R.; Seefeldt, L. C. Mechanism of Nitrogen Fixation by Nitrogenase: The Next Stage.
Chem. Rev. 2014, 114, 4041-62.

(56) Peterson, A. A.; Abild-Pedersen, F.; Studt, F.; Rossmeisl, J.; Nørskov, J. K. How Copper Catalyzes the Electroreduction of Carbon Dioxide into Hydrocarbon Fuels. Energy Environ. Sci. 2010, 3, 1311-1315.

(57) Skulason, E.; Bligaard, T.; Gudmundsdóttir, S.; Studt, F.; Rossmeisl, J.; Abild-Pedersen, F.; Vegge, T.; Jonsson, H.; Nørskov, J. K. A Theoretical Evaluation of Possible Transition Metal Electro-Catalysts for $\mathrm{N}_{2}$ Reduction. Phys. Chem. Chem. Phys. 2012, 14, 1235-1245.

(58) Ling, C.; Ouyang, Y.; Li, Q.; Bai, X.; Mao, X.; Du, A.; Wang, J. A General Two-Step Strategy-Based High-Throughput Screening of Single Atom Catalysts for Nitrogen Fixation. Small Methods 2018, 3, 1800376.

(59) Wang, S.; Shi, L.; Bai, X.; Li, Q.; Ling, C.; Wang, J. Highly Efficient Photo-/Electrocatalytic Reduction of Nitrogen into Ammonia by DualMetal Sites. ACS Cent. Sci. 2020, 6, 1762-1771.

(60) Qiu, W.; Xie, X. Y.; Qiu, J.; Fang, W. H.; Liang, R.; Ren, X.; Ji, X.; Cui, G.; Asiri, A. M.; Cui, G.; Tang, B.; Sun, X. High-Performance Artificial Nitrogen Fixation at Ambient Conditions Using a Metal-Free Electrocatalyst. Nat. Commun. 2018, 9, 3485.

(61) Ma, X. L.; Liu, J. C.; Xiao, H.; Li, J. Surface Single-Cluster Catalyst for $\mathrm{N}_{2}$-to- $\mathrm{NH}_{3}$ Thermal Conversion. J. Am. Chem. Soc. 2018, 140, 46-49.

(62) Ouyang, Y.; Shi, L.; Bai, X.; Li, Q.; Wang, J. Breaking Scaling Relations for Efficient $\mathrm{CO}_{2}$ Electrochemical Reduction through Dual-Atom Catalysts. Chem. Sci. 2020, 11, 1807-1813.

(63) Perdew, J. P.; Burke, K.; Ernzerhof, M. Generalized Gradient Approximation Made Simple. Phys. Rev. Lett. 1996, 77, 3865.

(64) Blöchl, P. E. Projector Augmented-Wave Method. Phys. Rev. B 1994, 50,17953

(65) Furthmüller, G. K. J., Efficient Iterative Schemes for Ab Initio TotalEnergy Calculations Using a Plane-Wave Basis Set. Phy. Rev. B 1996, 54, 11169-11186.

(66) Kresse, G.; Joubert, D. From Ultrasoft Pseudopotentials to the Projector Augmented-Wave Method. Phys. Rev. B 1999, 59, 1758-1775.

(67) Park, J.; Roling, L. T. Elucidating Energy Scaling between Atomic and Molecular Adsorbates in the Presence of Solvent. AIChE Journal 2020 66:e17036.

\section{TOC graphics}




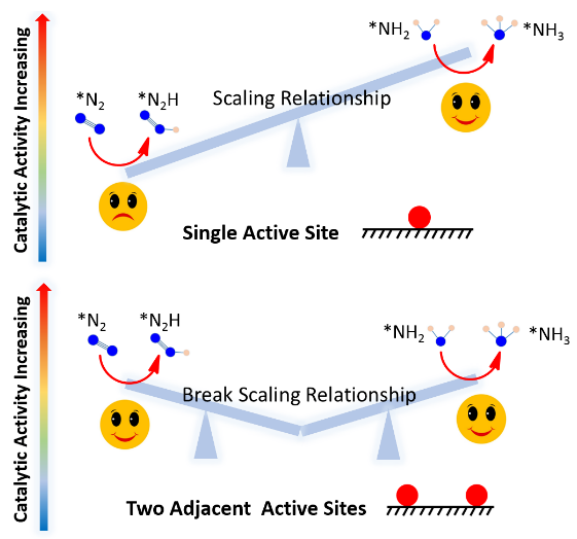

